\title{
Improved Upper Limit on Muonium to Antimuonium Conversion
}

\author{
R. Abela, ${ }^{1}$ J. Bagaturia, ${ }^{2}$ W. Bertl, ${ }^{1}$ R. Engfer,${ }^{3}$ B. Fischer von Weikersthal,${ }^{4}$ A. Großmann, ${ }^{4}$ V. W. Hughes ${ }^{5}$
} K. Jungmann, ${ }^{4}$ D. Kampmann, ${ }^{6}$ V. Karpuchin, ${ }^{7}$ I. Kisel, ${ }^{7}$ A. Klaas, ${ }^{16}$ S. Korenchenko, ${ }^{7}$ N. Kuchinsky, ${ }^{7}$ A. Leuschner, ${ }^{3}$ B. E. Matthias, ${ }^{4}$ R. Menz,${ }^{3}$ V. Meyer ${ }^{4}$ D. Mzavia, ${ }^{2}$ G. Otter,${ }^{6}$ T. Prokscha,${ }^{4}$ H. S. Pruys,${ }^{3}$ G. zu Putlitz,${ }^{4}$ W. Reichart, ${ }^{3}$ I. Reinhard, ${ }^{4}$ D. Renker, ${ }^{1}$ T. Sakhelashvilli, ${ }^{2}$ P. V. Schmidt, ${ }^{4}$ R. Seeliger, ${ }^{6}$ H. K. Walter, ${ }^{1}$ L. Willmann, ${ }^{4}$ and L. Zhang ${ }^{4}$

${ }^{1}$ Paul Scherrer Institut, CH-5232 Villigen PSI, Switzerland

${ }^{2}$ Tbilisi State University, GUS-380086 Tbilisi, Georgia

${ }^{3}$ Physik Institut, Universität Zürich, CH-8057 Zürich, Switzerland

${ }^{4}$ Physikalisches Institut der Universität Heidelberg, D-69120 Heidelberg, Germany

${ }^{5}$ Physics Department, Yale University, New Haven, Connecticut 06520

${ }^{6}$ Physik Institut B, Rheinisch-Westfälische Technische Hochschule Aachen, D-52056 Aachen, Germany

${ }^{7}$ Joint Institute of Nuclear Research, RU-141980 Dubna, Russia

(Received 13 June 1996)

\begin{abstract}
A new experiment has been set up at the Paul Scherrer Institut to search for muonium to antimuonium conversion. No event was found to fulfil the requested signature which consists of the coincident detection of both constituents of the antiatom in its decay. Assuming an effective $(V-A) \times(V-A)$ type interaction an improved upper limit is established for the conversion probability of $P_{M \bar{M}} \leq$ $8 \times 10^{-9}$ (90\% C.L.), which is almost 2 orders of magnitude lower compared to previous results and provides a sensitive test for theoretical extensions of the standard model. [S0031-9007(96)01128-3]
\end{abstract}

PACS numbers: 11.30.Fs, 11.30.Hv, 13.10.+q, 36.10.Dr

The hydrogenlike muonium atom $\left(M=\mu^{+} e^{-}\right)$consists of two leptons from different generations. Because of the close confinement of the bound state it offers excellent opportunities to study precisely the fundamental electron-muon interaction as described in standard theory and to search sensitively for additional so far unknown interactions between these two particles. A spontaneous conversion of muonium into antimuonium $\left(\bar{M}=\mu^{-} e^{+}\right)$ would violate additive lepton family number conservation by two units. In the standard model, which is a very successful description of experimental particle physics, this process is not provided like other decays which are searched for, e.g., the muon decay modes $\mu^{+} \rightarrow e^{+} \nu_{\mu} \bar{\nu}_{e}$ [1], $\mu \rightarrow e \gamma$ [2], $\mu \rightarrow e e e$ [3], and $\mu \rightarrow e$ conversion [4]. However, in the framework of many speculative theories, which try to extend the standard model in order to explain further some of the features like parity violation in weak interaction or the particle mass spectra, lepton number violation appears to be natural and muonium to antimuonium conversion is an essential part in several of those models (Table I) [5-10]. The coupling constant $G_{M \bar{M}}$ in an effective four fermion interaction [11] could be as large as the present experimental limit of $G_{M} \bar{M} \leq 0.16 G_{F}(90 \%$ C.L.) established at LAMPF in Los Alamos [12], or the bound of $G_{M} \bar{M} \leq 0.14 G_{F}(90 \%$ C.L.) [13] very recently proposed from an experiment at the Phasotron in Dubna, Russia, where $G_{F}$ is the Fermi coupling constant of the weak interaction. In particular, in the framework of minimal left-right symmetric theory a lower bound has been predicted with the assumption of a muon neutrino mass $m_{\mu_{\nu}}$ larger than $35 \mathrm{keV} / c^{2}$.

At the Paul Scherrer Institut (PSI) in Villigen, Switzerland, a new experiment has been set up (Fig. 1), which utilizes the powerful signature for a conversion developed in the recent LAMPF experiment [12]. It requires the coincident identification of both constituents of the antiatom,

TABLE I. Muonium to antimuonium conversion is allowed in some speculative extensions to the standard model. The lower limit given for minimal left-right symmetry corresponds to a muon neutrino mass limit of $m_{\nu \mu} \leq 160 \mathrm{keV} / c^{2}[21]$.

\begin{tabular}{|c|c|c|}
\hline Model & Limit & Ref. \\
\hline $\begin{array}{l}\text { Minimal left-right symmetry with extended Higgs sector; conversion } \\
\text { through exchange of doubly charged Higgs boson } \Delta^{++}\end{array}$ & $G_{M \bar{M}} \geq 2 \times 10^{-4} G_{F}$ & [5] \\
\hline $\begin{array}{l}Z_{8} \text { model with fourth generation of heavy leptons and radiative mass } \\
\text { generation in leptonic sector; conversion through exchange of neutral } \\
\text { scaler boson } \Phi^{0}\end{array}$ & $G_{M \bar{M}} \leq 10^{-2} G_{F}$ & [7] \\
\hline $\begin{array}{l}\text { Supersymmetric model with broken } R \text { parity; conversion through } \\
\text { exchange of } \tau \text {-sneutrino } \tilde{\nu}_{\tau}\end{array}$ & $G_{M \bar{M}} \leq 10^{-2} G_{F}$ & {$[8,9]$} \\
\hline $\begin{array}{l}\text { GUT models; conversion through exchange of doubly charged dileptonic } \\
\text { gauge boson } X^{ \pm \pm}\end{array}$ & $G_{M \bar{M}} \leq 0.13 G_{F}$ & {$[10]$} \\
\hline
\end{tabular}


$\mu^{-}$and $e^{+}$, in its decay. Since a possible conversion is suppressed in matter mainly due to the removal of degeneracy between muonium and antimuonium [11,14], a sensitive experiment needs the atoms to be in vacuum. This experiment utilizes the most efficient method known to date to provide muonium in vacuum: Positive muons from a continuous beam of momentum $p=21 \mathrm{MeV} / c$ and momentum bite $\Delta p / p=6 \%$ at a typical beam rate of $8 \times 10^{5} \mathrm{~s}^{-1}$ are stopped in a silicon dioxide $\left(\mathrm{SiO}_{2}\right)$ powder target of thickness $8 \mathrm{mg} / \mathrm{cm}^{2}$. About $60 \%$ of the muons form muonium atoms [15], some of which leave the target through its surface into the surrounding vacuum with thermal energies, corresponding to a velocity of 7.4(1) $\mathrm{mm} / \mu \mathrm{s} \mathrm{[16].}$

Electrons from the decay $\mu^{-} \rightarrow e^{-}+\overline{\nu_{e}}+\nu_{\mu}$ of an antimuonium atom have an energy spectrum ranging up to $53 \mathrm{MeV}$. They can be observed in a magnetic spectrometer consisting of five cylindrical proportional chambers surrounded by a hodoscope of 64 plastic scintillator stripes. Three of the wire chambers are equipped with two planes of helical cathode stripes allowing one to measure both, angular and axial, coordinates [3]. A solenoidal magnet provides an axial magnetic field of $0.1 \mathrm{~T}$ for measuring particle momenta and identification of their sign of charge. After the decay of the antiatoms the positrons from their atomic shells are left behind with an average kinetic energy of $R_{\mu}=13.5 \mathrm{eV}$ [17]. These positrons can be accelerated parallel to the magnetic flux lines up to typically $8 \mathrm{kV}$ in a two stage electrostatic device and guided in a magnetic field of $B=0.1 \mathrm{~T}$ onto a position sensitive microchannel plate detector (MCP) with resistive anode readout [18]. The transport system consists of two

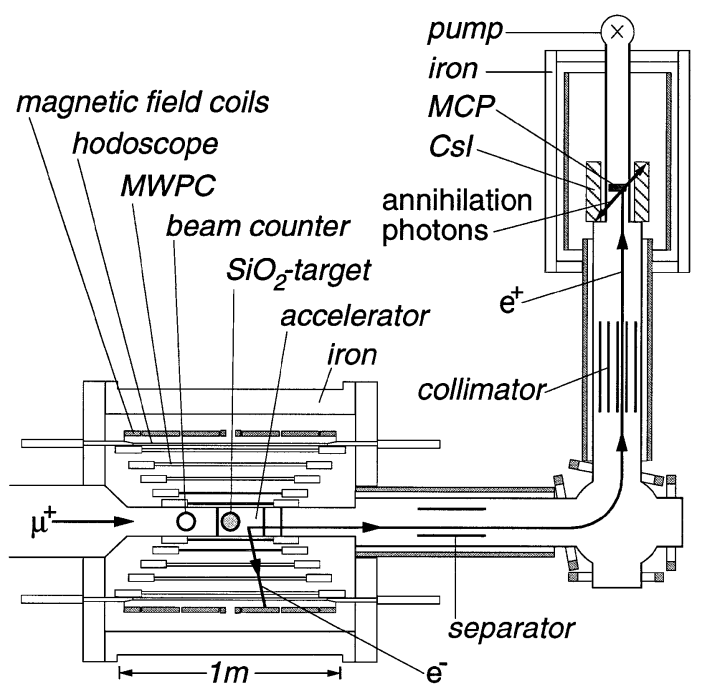

FIG. 1. Top view of the new apparatus at PSI to search for muonium-antimuonium conversion. The observation of an energetic electron from the $\mu^{-}$decay in the antiatom in a magnetic spectrometer is required in coincidence with the detection of the positron, which is left behind from the atomic shell of the antiatom, on a MCP and at least one annihilation photon in a CsI calorimeter. straight sections of $1.5 \mathrm{~m}$ in length with a $90^{\circ}$ bend of radius $35 \mathrm{~cm}$ between them (Fig. 1), and it is momentum selective with a transmission maximum for $8 \mathrm{keV}$ positrons. An electric field orthogonal to the magnetic field applied in the first section displaces charged particles by a distance depending on their velocity and removes slow ions or muons. Particles of longitudinal momenta exceeding $750 \mathrm{keV} / c$ cannot follow adiabatically the magnetic flux lines in the bend region. They gain transverse momenta above $135 \mathrm{keV} / \mathrm{c}$ resulting in gyration radii larger than $4.5 \mathrm{~mm}$, and they are stopped in a collimator of length $40 \mathrm{~cm}$ made from copper foils of thickness $1 \mathrm{~mm}$ with a spacing of $10 \mathrm{~mm}$. The magnetic field gradient causes, in addition, a drift orthogonal to both the field and its gradient for charged particles proportional to their momentum.

The positron track can be retraced from the position measured on the MCP detector to the target region with an accuracy of $1 \mathrm{~mm}$. The decay position of the atom can be determined from the vertex with the track of the high energetic particle in the magnetic spectrometer. In addition, the detection of at least one of the $511 \mathrm{keV}$ photons from positron annihilation on the MCP in a 12-fold segmented pure CsI crystal detector surrounding the MCP is required as a part of the signature. The crystal detector was calibrated using positrons from a ${ }^{22} \mathrm{Na}$ source. The acceptance for at least one of the annihilation photons has been measured to be 79(4)\%. The time resolution of the device is 5.4(3) ns (FWHM).

The new experiment was designed to provide as high as possible symmetry in the detection of muonium and antimuonium decays in order to minimize the influence of systematic uncertainties arising from corrections for efficiencies and acceptances of various detector components. In the course of data acquisition the polarity of all electric and magnetic fields were reversed regularly every $4 \mathrm{~h}$ for a duration of $20 \mathrm{~min}$ to monitor the muonium production and for checking the calibration of the detector components and the transport system parameters. In this case energetic positrons and atomic electrons from muonium decays were detected. For a time of flight measurement the time interval between the arrival of a muon signaled by the beam counter and the detection of its decay positron in the hodoscope was recorded at low beam rates. On average every second day the $\mathrm{SiO}_{2}$ targets were replaced to compensate for an observed decrease of the muonium yield. The time of flight for the electrons from the atomic shell was determined to be 75.8(1) ns by observing muonium atom decays at $8 \mathrm{kV}$ acceleration voltage. The signal width of 5.7(1) ns (FWHM) allows one to apply a $20 \mathrm{~ns}$ narrow coincidence time window in the event signature.

The evaluation of the muonium production data is based on a model established in independent dedicated experiments [16], which assumes that the atoms are produced inside the $\mathrm{SiO}_{2}$ powder at the positions given by the stopping distribution of the muons. A 
one-dimensional diffusion process describes the escape of the muonium atoms into vacuum where their velocities follow a Maxwell-Boltzmann distribution. The number of atoms in the fiducial volume has been extracted from two different projections. First, the distribution of time intervals between a beam counter signal from the incoming muon and the detection of the atomic electron on the MCP yields the fraction of muonium in vacuum [Fig. 2(a)]. Second, this quantity has been derived by a two-dimensional maximum likelihood fit to the distribution of the atoms decay positions downstream of the target and their respective time of flight [Fig. 2(b)]. Both methods agree within their error margins and yield an average fraction of observed muonium atoms of $3.1(2) \times 10^{-3}$ per incoming muon. This figure includes all detection efficiencies, i.e., the solid angle of the magnetic spectrometer $(0.71 \times 4 \pi)$, the track reconstruction efficiency (0.85), the MCP detection efficiency [0.16(2)], and the geometric acceptance of the transport system for electrons $(0.80)$. The average fraction of muonium atoms in vacuum per incoming muon amounts to $5.7(8) \%$, in good agreement with results from the earlier dedicated studies of muonium production [16].

(a)
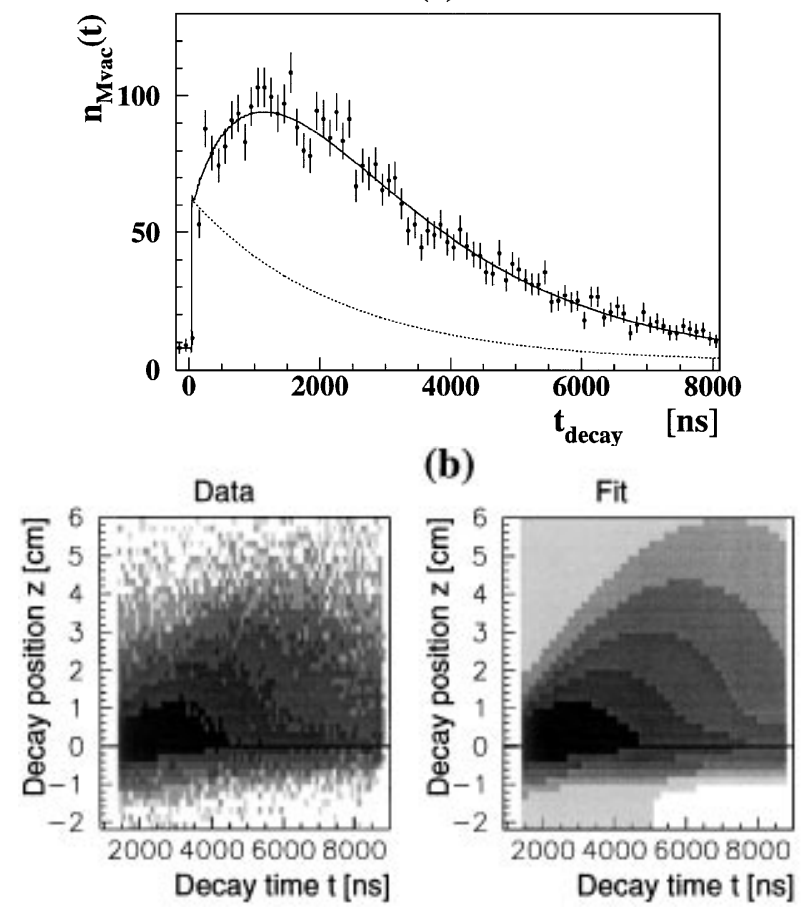

FIG. 2. (a) The yield of muonium atoms in vacuum can be obtained from the distribution of time intervals between the incoming muon detected by the beam counter and the observation of the decay of an atom in vacuum. The dashed line represents an exponentially decaying background. (b) An independent two-dimensional fit to the observed muon decays in the vacuum region downstream of the $\mathrm{SiO}_{2}$ target as a function of the time and position gives the same muonium fraction. Logarithmic contours are displayed.
The effective measurement time for the search for antimuonium was $210 \mathrm{~h}$ during which $1.4(1) \times 10^{9}$ muonium atoms were in the fiducial volume of diameter $9 \mathrm{~cm}$ and length $10 \mathrm{~cm}$. Data were recorded at acceleration voltages between $2-10 \mathrm{kV}$ to allow for systematic studies of the transport system. No decay of an antimuonium atom was found. There is no entry in a $20 \mathrm{~ns}$ time window around the expected time of flight in the corresponding distribution (Fig. 3). The apparent structure around $t_{\mathrm{TOF}}-t_{\text {expected }}=-50 \mathrm{~ns}$ corresponds to the allowed rare decay mode $\mu^{+} \rightarrow e^{+} e^{+} e^{-} \nu_{e} \overline{\nu_{\mu}}$ in which one of the positrons is released with low kinetic energy, while the electron is detected in the magnetic spectrometer and partly to Bhabha scattering. Because of their significantly higher initial momenta positrons from these processes arrive at earlier times at the MCP and can be clearly distinguished from possible antimuonium decays. In addition, for those events no decay vertex can be reconstructed inside the fiducial volume, because of the strong deflection of high energetic positrons in the bend of the transport system. The background due to these processes in the search for antimuonium has been estimated from Monte Carlo simulations to be below $10^{-14}$ per incoming $\mu^{+}$.

Taking into account the detection efficiency for the annihilation photon in the CsI detector and corrections for the finite fiducial volume and finite observation time [0.74(2)],
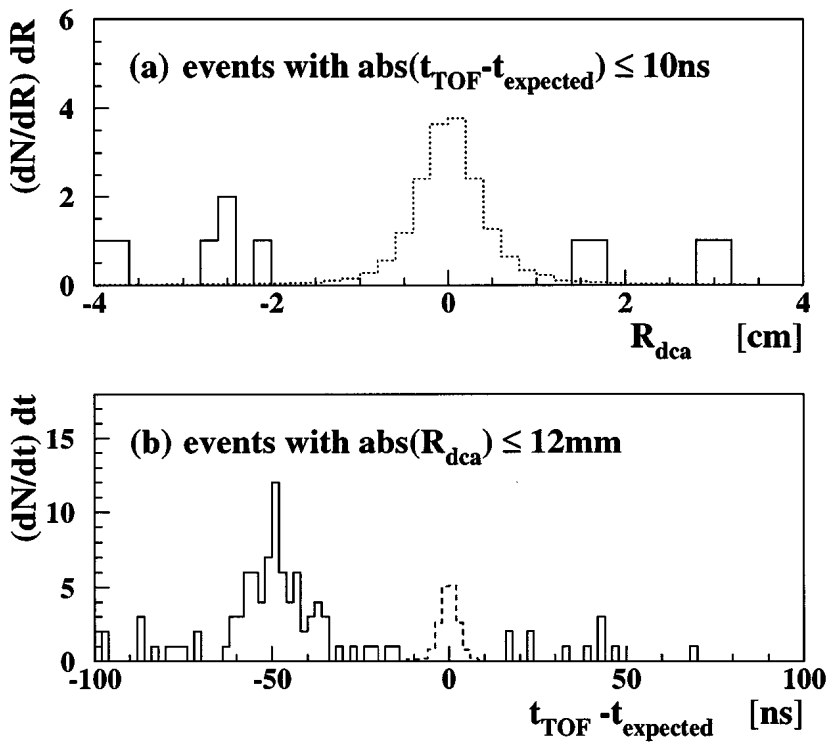

FIG. 3. The number of events with identified energetic electron and positron as a function of (a) the distance of closest approach $R_{\mathrm{dca}}$ between the electron track in the magnetic spectrometer and the back projection of the position measured at the MCP and (b) the difference of the positrons time of flight $t_{\mathrm{TOF}}$ to the expected arrival time $t_{\text {expected }}$. The signal at earlier times corresponds to the allowed decay channel $\mu \rightarrow 3 e 2 \nu$ and Bhabha scattering. It is smeared out due to different acceleration voltages used. No event satisfied the required coincidence signature. The dashed curves correspond to simulated signals scaled from the relevant distributions recorded while monitoring the muonium production for $G_{M \bar{M}}=0.05 G_{F}$. 
an upper limit can be set on the conversion probability in a $0.1 \mathrm{~T}$ magnetic field of $P_{M \bar{M}}(0.1 \mathrm{~T}) \leq 2.8 \times 10^{-9}$ (90\% C.L.). The conversion is suppressed in external magnetic fields due to the removal of the degeneracy of the energy levels in muonium and antimuonium atoms and has been calculated recently for different types of interactions [19,20]. For $(V \pm A) \times(V \pm A)$ interactions, the conversion probability at a magnetic field of $0.1 \mathrm{~T}$ is suppressed to $35 \%$ of the zero field value, while for a $(V-A) \times(V+A)$ interaction it is reduced to only $77.6 \%$. In case of a future observation of muoniumantimuonium conversion the coupling type could be determined by a measurement of the ratio of conversion probabilities at different magnetic field strengths. For an effective $(V \pm A) \times(V \pm A)$ interaction, where the zero field probability $P_{M \bar{M}}(0 \mathrm{~T})$ is related to the coupling constant $G_{M \bar{M}}$ through

$$
G_{M \bar{M}}=G_{F} \sqrt{\frac{P_{M \bar{M}}(0 \mathrm{~T})}{2.56 \times 10^{-5}}},
$$

we have $P_{M} \bar{M}(0 \mathrm{~T}) \leq 8 \times 10^{-9}$ (90\% C.L.) and an upper limit of $G_{M \bar{M}} \leq 1.8 \times 10^{-2} G_{F}(90 \%$ C.L.). In GUT models where a muonium-antimuonium conversion could be mediated via the exchange of a dileptonic gauge boson $X^{ \pm \pm}$a tight new mass limit of $M_{X^{ \pm \pm}} / g_{3 l}>1.1 \mathrm{TeV} / c^{2}$ (90\% C.L.) can be extracted where $g_{3 l}$ depends on the particular symmetry and is of order unity. With the limit established in this experiment models with dilepton exchange [10] as well as models with heavy leptons and radiative generation of leptons masses appear less attractive [7].

With improved detectors for $8 \mathrm{keV}$ positrons which are now available with 4 times enhanced efficiency [18] and by utilizing the beam line $\pi$ E5 at PSI with 10 times higher muon fluxes compared to the $\pi \mathrm{E} 3$ area used for this experiment we expect another significant improvement in the sensitivity on the conversion probability in the future which could provide a more stringent test for speculative extensions to the standard model, in particular left-right symmetric models predicting a lower bound on $G_{M \bar{M}}$.

This work is supported in part by the German BMBF, the Swiss Nationalfond, the Russian FFR, and a NATO research grant. The collaboration is grateful to the staff at PSI for providing excellent working conditions in a friendly atmosphere.

[1] S. Freedman et al., Phys. Rev. D 47, 811 (1993).

[2] R. D. Bolton et al., Phys. Rev. D 38, 2077 (1988).

[3] W. Bertl et al., Nucl. Phys. B260, 1 (1985).

[4] W. Honecker et al., Phys. Rev. Lett. 76, 200 (1996).

[5] P. Herczeg and R. N. Mohapatra, Phys. Rev. Lett. 69, 2475 (1992).

[6] A. Halprin, Phys. Rev. Lett. 48, 1313 (1982).

[7] G. G. Wong and W.S. Hou, Phys. Rev. D 50, R2962 (1994); W.S. Hou and G. G. Wong, Phys. Rev. D 53, 1537 (1996).

[8] R. N. Mohapatra, Z. Phys. C 56, 117 (1992).

[9] A. Halprin and A. Masiero, Phys. Rev. D 48, 2987 (1993).

[10] H. Fujii et al., Phys. Rev. D 49, 559 (1994).

[11] G. Feinberg and S. Weinberg, Phys. Rev. 123, 1439 (1961).

[12] B. E. Matthias et al., Phys. Rev. Lett. 66, 2716 (1991).

[13] V. A. Gordeev et al., JETP Lett. 59, 589 (1994).

[14] D. L. Morgan, Jr. and V. W. Hughes, Phys. Rev. D 2, 1389 (1970); Phys. Rev. A 7, 1811 (1973).

[15] R. F. Kiefl et al., Phys. Rev. B 26, 2432 (1982).

[16] K. Woodle et al., Z. Phys. D 9, 59 (1988); see also A. C. Janissen et al., Phys. Rev. A 42, 161 (1990).

[17] L. Chatterjee et al., Phys. Rev. D 46, 46 (1992).

[18] P. V. Schmidt et al., Nucl. Instrum. Methods Phys. Res., Sect. A (to be published).

[19] G. G. Wong and W. S. Hou, Phys. Lett. B 357, 145 (1995).

[20] K. Horikawa and K. Sasaki, Phys. Rev. D 53, 560 (1996).

[21] K. Assamagan et al., Phys. Lett. B 335, 231 (1994). 